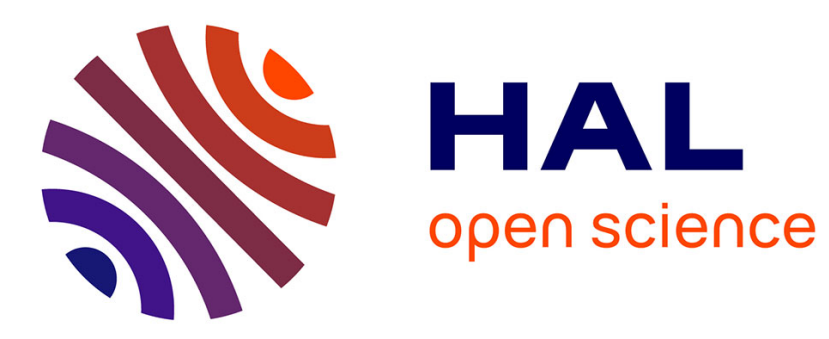

\title{
Assessing neuromuscular mechanisms in human-exoskeleton interaction
}

Nahéma Sylla, Vincent Bonnet, Gentiane Venture, Nasser Armande, Philippe Fraisse

\section{- To cite this version:}

Nahéma Sylla, Vincent Bonnet, Gentiane Venture, Nasser Armande, Philippe Fraisse. Assessing neuromuscular mechanisms in human-exoskeleton interaction. EMBC: Engineering in Medicine and Biology Conference, Aug 2014, Chicago, IL, United States. pp.1210-1213, 10.1109/EMBC.2014.6943814 . lirmm-01111277

\section{HAL Id: lirmm-01111277 https://hal-lirmm.ccsd.cnrs.fr/lirmm-01111277}

Submitted on 30 Jan 2015

HAL is a multi-disciplinary open access archive for the deposit and dissemination of scientific research documents, whether they are published or not. The documents may come from teaching and research institutions in France or abroad, or from public or private research centers.
L'archive ouverte pluridisciplinaire HAL, est destinée au dépôt et à la diffusion de documents scientifiques de niveau recherche, publiés ou non, émanant des établissements d'enseignement et de recherche français ou étrangers, des laboratoires publics ou privés. 


\title{
Assessing Neuromuscular Mechanisms in Human-Exoskeleton Interaction
}

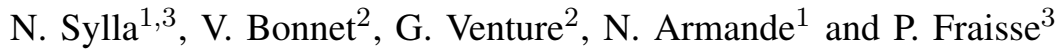

\begin{abstract}
In this study, we propose to evaluate a 7 DOF exoskeleton in terms of motion control. Using criteria from the human motor control literature, inverse optimization was performed to assess an industrial screwing movement. The results of our study show that the hybrid composition of the free arm movement was accurately determined. At contrary, when wearing the exoskeleton, which produces an arbitrary determined torque compensation, the motion is different from the naturally adopted one. This study is part of the evaluation and comprehension of the complex neuromuscular mechanism resulting in wearing an exoskeleton several hours per day for industrial tasks assistance.
\end{abstract}

\section{INTRODUCTION}

In car assembly lines, several operations are performed by workers because of dexterity and high precision requirements. However, these manual operations can lead to musculo-skeletal disorders for workers population because of awkward adopted postures and repeated motions performed during very short cycle time [1]. In PSA Peugeot Citroen factories, one operation consists of screwing pieces in cars located above workers's head, with a task time of one car per minute. High solicitations of upper limbs and motion fastness requirements make the operation very challenging in terms of occupational health. Hence, the usage of an upper-limb exoskeleton might be a possible solution to assist workers dedicated to this task. Upper-limb exoskeletons were mainly designed for rehabilitation of stroke patients or for helping people in performing painful tasks. Kiguchiet al. designed a 3 degrees-of-freedom (DOF) exoskeleton for only shoulder and elbow motions [2]. Later, Gopura et al. designed a 7 DOF arm exoskeleton called SUEFUL-7 [3], which uses realtime control method based on skin surface electromyographic (EMG) signals. However, the exoskeleton presented limited shoulder movements while large workspaces were provided for elbow and wrist movements. The HAL whole body exoskeleton [4] uses also a similar method for movement assistance. Although its effectiveness, the usage of EMG requires accurate musculo-skeletal system modelling and individual calibration for each user, making it hardly suitable

\footnotetext{
${ }^{1}$ PSA Peugeot Citroen Research and Development Department, Centre Technique de Vlizy, Route de Gisy, 78140 Velizy Villacoublay, France, khadijanahema.sylla@mpsa.com, nahid.armande@mpsa.com

${ }^{2} \mathrm{GV}$ laboratory, Dpt. of Mechanical Systems Engineering, Tokyo University of Agriculture and Technology, Tokyo University, Japan vincent.bonnet@gmail.com, venturedcc.tuat.ac.jp

${ }^{3}$ LIRMM (Montpellier laboratory of Informatics, Robotics and Micro-electronics), 161 rue Ada, 34392 Montpellier Cedex 5, France fraissedirmm.fr
}

for industrial context. Unlike EMG-based control methods, the patented force-position control method developed for ABLE exoskeleton does not require calibration and force sensor [5] [6]. ABLE was retained for its backdrivability which allows to use it as an arm weight compensation device. In order to use this type of exoskeleton in PSA car assembly lines, it is necessary to assess its performances in terms of effort compensation, human movements coordination, and neuromuscular mechanisms. A previous study [7] was performed to assess the use of ABLE 7 axes exoskeleton in this context. Results show a clear reduction of joint torques and a slight modifications of joint angle trajectories. Regarding the complexity of the human musculoskeletal system, the influence of the exoskeleton in human movements remains difficult to assess with a classic biomechanical analysis. The approach proposed in this paper analyses the human-exoskeleton interaction in terms of neuromuscular mechanisms. Human motor control researchers have been investigating for several years the neuromuscular mechanisms underlying the motion. From their extensive debates, it appears that there is a consensus stating that the central nervous system determines in an optimal way the motion strategy among several solutions before sending the information to the musculoskeletal system [8]. Computational models, based on representative biomechanical models were used to minimize a given criterion, also called a cost-function, to determine the optimal set of trajectories. However, the choice of this criterion is not trivial because of kinematic, dynamic and actuation redundancy of the human musculoskeletal system. Several criteria such as minimum jerk [9], minimum torque change [10], minimum energy [11], minimum work [12], or minimum variance [13] have been proposed for multi-joints arm movements. However, from this literature no consensus emerges and it appears that each of these optimal criteria accounts for a feature of the motion depending on the considered task. The use of hybrid cost-functions was then introduce to determine the optimal motion strategy used by the central nervous system. An inverse optimization algorithm proposed by Botasso et $a l$. allows to predict movements of multibody neuromuscular systems [14]. The method has been validated for arm and leg models. However, it uses simple cost function search spaces, and the algorithm was not investigated for complex tasks. The inverse optimal framework proposed by Mombaur et al. [15] determines optimality criteria that produce natural paths for human locomotion. In this study, the human body was considered as a single point and experiments were performed in a $2 \mathrm{D}$ plane, which simplified significantly the inverse 
optimization problem. Later Berret et al. proposed a similar approach to obtain the best cost function which characterizes a reaching-a-bar movement [16]. However, this interesting and well documented study focuses on a simple planar task with a poorly constrained paradigm. In this context, this study proposes to assess the control law of ABLE exoskeleton at a neuromuscular level. Based on studies presented in [15] and [16], the inverse optimization control is use to determine biological criteria involved when the movement is performed with the exoskeleton.

\section{DESCRIPTION OF ABLE EXOSKELETON}

ABLE is a right mono-arm exoskeleton with 7 DOF allowing to follow human movements in a large workspace [6]. Its patented actuation system presented in details in the literature allows hybrid force and position control without requiring any force sensor [5]. The ABLE controller is based on two major functions: the friction compensation, and the weight compensation. The dry friction compensation $\left(\Gamma_{f}\right)$ is the key for the transparency and is modelled in the controller by:

$$
\Gamma_{f}=\tanh (\epsilon \cdot \omega) \cdot f,
$$

where $f$ is the static friction, $\omega$ the motor speed, and $\tanh (\epsilon . \omega)$ the smoothing function, $\epsilon$ being the slope at the origin. Gravity compensation level is given by the value of the effort exerted on the $k^{t h}$ axis $\left(\Gamma_{f}\right)$, defined in the following expression:

$$
\Gamma_{k}=\left(m_{x_{k}} \cos \theta+m_{y_{k}} \sin \theta\right) \cdot g
$$

where $g$ is the gravity acceleration, $\theta$ is the angle of the exoskeleton, $m_{x}$ and $m_{y}$ are contributions of the mass of $\mathrm{x}$ and $y$ axis respectively, times the distance of the center of mass:

$$
m_{x_{k}}=x_{g_{k}} * m_{k}
$$

In this study, the gravity compensation is used to reduce users' effort with different levels of compensation, obtained by increasing $m_{x_{3}}$ parameter which corresponds to the shoulder joint in the sagittal plane.

\section{EXPERIMENTATION}

\section{A. Measurements and modelling}

Eight right-handed volunteers (size: 1.65 to $1.75 \mathrm{~m}$; weight: 50 to $68 \mathrm{~kg}$; age: 23 to 30 years old) not familiar with the task were asked to reproduce the typical screwing movement. With an initial standing position with both hands along the body side, subjects have to reach a target point located 2 meters above the ground. The screwing movement was performed by each subject in four different situations. During the first one (Trial 1), the movement was carried out without the exoskeleton while for the followings, subjects were assisted by the exoskeleton with increasing levels of compensation (Trial 2: $m_{x_{3}}=1.335 \mathrm{~kg} . \mathrm{m}$, Trial 3: $m_{x_{3}}=1.635$ kg.m, Trial 4: $m_{x_{3}}=1.935$ kg.m). A motion capture system (6 MX cameras, VICON, 100Hz) in combination with a set of 38 retro-reflective markers, placed on anatomical

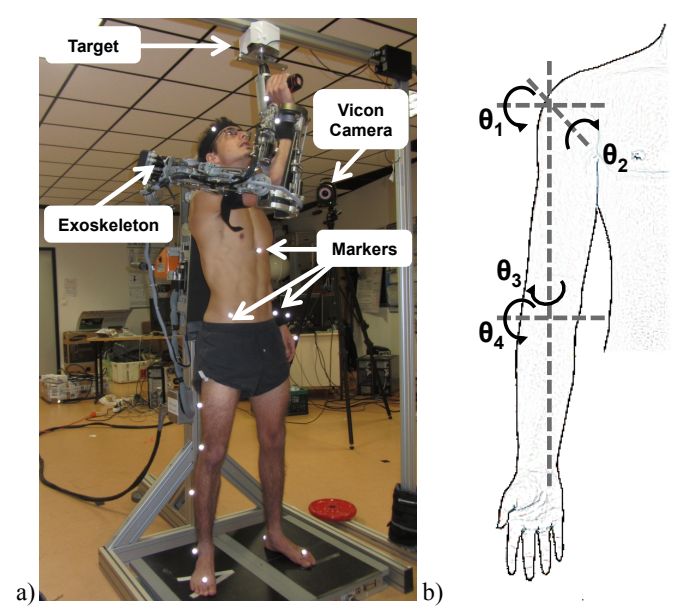

Fig. 1. Investigated screwing movement carried out with the exoskeleton (a), human arm model used in the study (b)

landmarks in accordance with the Plugin-Gait whole body marker template (Vicon Motion Systems), was used to record 3D kinematic quantities (see Fig. 1a). As expected, low values of right wrist angles were measured, allowing us to neglect wrist DOF in the retained arm kinematic model, which presents 4 DOF represented in Fig. 1b. To analyse subjects energy expenditure during the movement, mean of joint torques $\left(\Gamma_{1}, \Gamma_{2}, \Gamma_{3}\right.$, and $\left.\Gamma_{4}\right)$ were compared in the four situations as in a previous study [7].

\section{B. Optimization Processing}

The proposed approach is to solve the so-called inverse optimization problem. A first inner optimization process finds joint trajectories that minimize a weighted hybrid cost function $C$, composed of several biologically plausible cost functions [16]:

$$
C=\sum_{i=1}^{6} \alpha_{i} c_{i},
$$

where $\alpha_{i}$ are the positive weights that define the contribution of every cost function $\mathrm{Ci}$, listed in Table I. A second outer optimization is used to determine the optimal weights that will minimize the difference between measured quantities and the output of the first inner optimization. This difference is evaluated here with the root mean square error (RMSE) between estimated $\left(\theta_{\text {est }}\right)$ and measured $\left(\theta_{m e s}\right)$ joint angles. Human angular limits defined in the literature [17] and the right hand final position are given here as constraints. The first optimisation process, which determines the optimal set of weights $\alpha$ is performed with a genetic algorithm [18] due to the large scale of the optimization problem. The second one, which determines optimal joint angle trajectories is performed using a gradient based non linear optimization algorithm [19].

Resulting joint trajectories and cost-functions are evaluated in the four situations to assess the influence of ABLE exoskeleton in terms of musculo-skeletal mechanisms. 
TABLE I

COST FUNCTION USED IN THE STUDY [16]

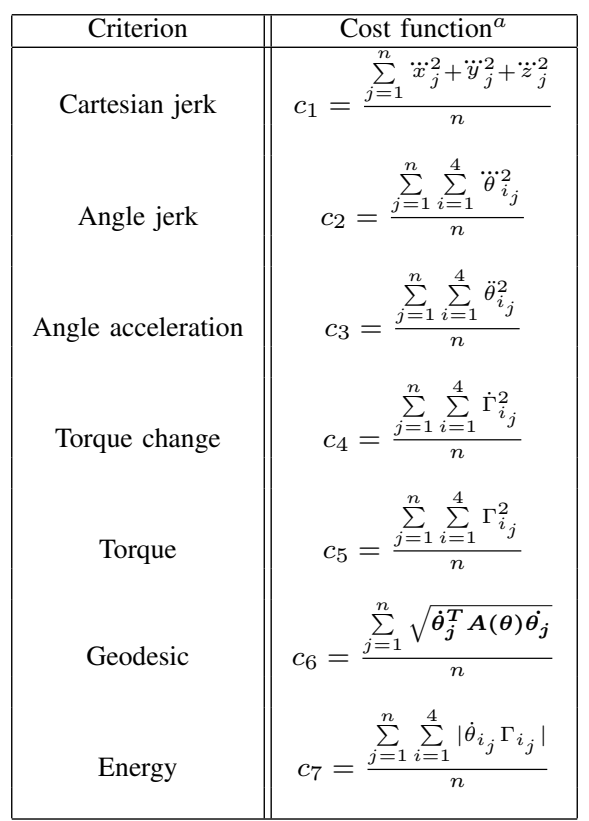

a. $n$ is the length of joint angles and positions variables
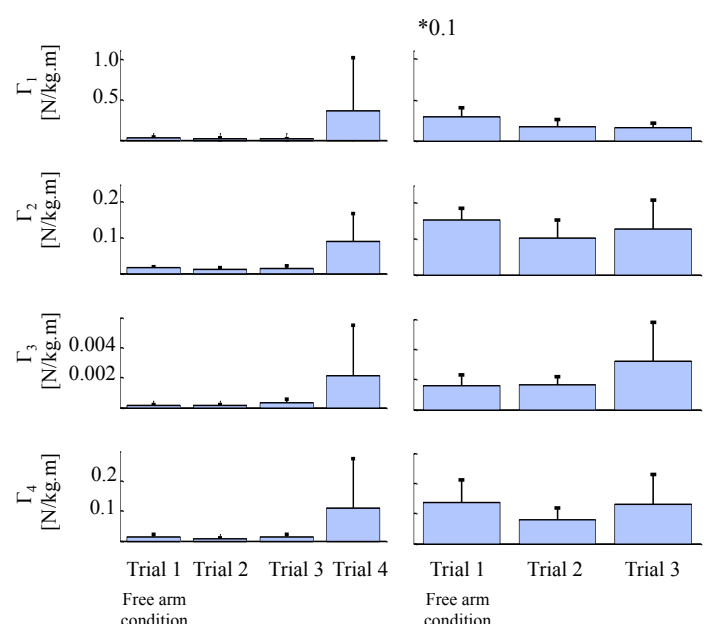

a)

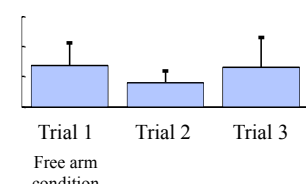

b)

Fig. 2. Mean (standard deviations) of measured joint torques for trials 1 to 4 (a) and for trial 1 to 3 (b)

\section{RESULTS}

\section{A. Human experiments}

Mean of joint torques, normalized by subjects' weight and height are represented in Fig. 2. From Fig. 2a, we can see that human joint torques increase significantly in the last situation. In this configuration the compensation level is not adapted to subjects, making them following the very artificial trajectory imposed by the exoskeleton [7]. Excluding this case (see Fig. 2b), when comparing joint torques in Trial 1 (i.e. in the free arm condition) and the others, joint torques decrease particularly for shoulder flexion/extension that performs the main part of the movement. From this figure we can also see that $\Gamma_{3}$ increases in Trial 3. However, the low value of $\Gamma_{3}$ is negligible, and does not influence the arm global effort. From a purely mechanical viewpoint, the reduction of joint torques justifies here the supply of the exoskeleton.

\section{B. Inverse optimization}

When analysing the results obtained with the inverse optimization process, represented here for a typical subject (Fig. 3 and 4), we can see a good fitting between the predicted trajectory of the model and the measured one in the freearm condition (Trial 1), indicated by low RMSE between joint angles. In this case, the subject minimizes the geodesic criterion, which is here an image of the kinetic energy, and the energy criterion. When the movement is performed with the exoskeleton, more important RMSE are reported (Fig. 3 and 5) and cost functions vary from a trial to an other (Fig. 4), indicating that the proposed model, based on six of the most common cost-functions from the literature accounting for natural grasping-reaching like motion, is not able to predict the observed strategy. From this statement two conclusions can be made. First, the motion is not natural and the exoskeleton compensation, based on a static compensative torque, is not appropriate. This means that the movement is not led by the human, but by the exoskeleton. An other conclusion might be that the subject is exploring other strategies involving the interaction of the exoskeleton. This can explain the increase of variability observed in human [7]. It is possible that after a certain adaptation time the subject was able to integrate the exoskeleton in his perception scheme, i.e. in the new optimal strategy. In any case, we believe that the synthesis of a new optimal control law based on the result obtained during the free-arm condition should improve the joint torque compensation, user's perception, and minimise the adaptation time. The idea would be to propose a personalised compensation relatively to the subject's anthropometric measurements being the results of the minimisation of the identified hybrid cost function (43\% energy and $57 \%$ of the geodesic cost function).

\section{CONCLUSION}

In this study a method is proposed to asses the use of an exoskeleton for an industrial screwing task at two levels. From a mechanical viewpoint, subjects efforts are compensated by the joint torque reduction provided by the exoskeleton. In terms of neuromuscular mechanisms, differences are reported between predicted and measured joint angles, and the hybrid cost function varies relatively to the compensation level. Although the effectiveness of the method for free-arm movements, more investigations have to be performed with experimented subjects during prolonged period of time to conclude about the cost function variations when the movement is performed with the exoskeleton. 


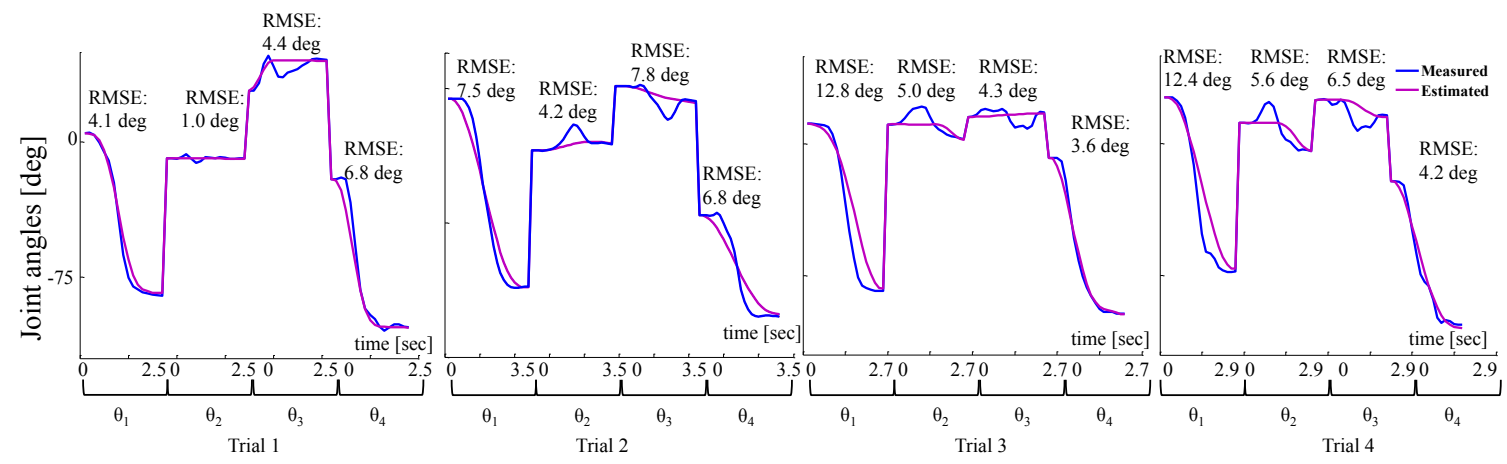

Fig. 3. Measured and estimated joint angles and their corresponding RMSE for all trials

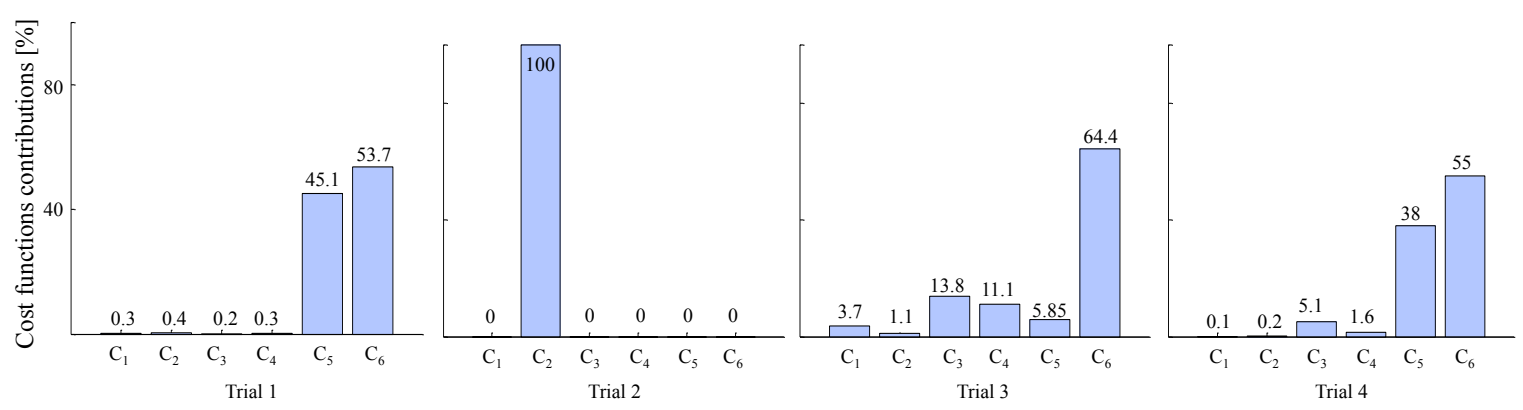

Fig. 4. Cost function contributions for all trials

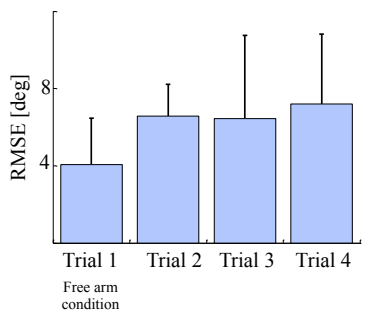

Fig. 5. Mean (standard deviations) of joint angles RMSE for all trials

\section{REFERENCES}

[1] B. P. Bernard et al., "Musculoskeletal disorders and workplace factors: a critical review of epidemiologic evidence for work-related musculoskeletal disorders of the neck, upper extremity, and low back," NIOSH, 1997.

[2] K. Kiguchi, T. Tanaka, K. Watanabe, and T. Fukuda, "Exoskeleton for human upper-limb motion support," in IEEE Int. Conf.on Robotics and Automation (ICRA), 2003. Proceedings., vol. 2, pp. 2206-2211, IEEE, 2003.

[3] R. A. R. C. Gopura, K. Kiguchi, and Y. Li, "Sueful-7: a 7dof upper-limb exoskeleton robot with muscle-model-oriented emg-based control," in IEEE/RSJ Int. Conf. on Intelligent Robots and Systems (IROS), pp. 1126-1131, IEEE, 2009.

[4] Y. Sankai, "Leading edge of cybernics: Robot suit hal," in SICEICASE, 2006. International Joint Conference, pp. P-1, IEEE, 2006.

[5] P. Garrec, J. Friconneau, Y. Measson, and Y. Perrot, "Able, an innovative transparent exoskeleton for the upper-limb," in IEEE/RSJ Int. Conf. on Intelligent Robots and Systems (IROS), pp. 1483-1488, IEEE, 2008.

[6] N. Jarassé, Contributions à l'exploitation d'exosquelettes actifs pour la rééducation neuromotrice. $\mathrm{PhD}$ thesis, Université Pierre et Marie Curie-Paris VI, 2010.

[7] N. Sylla, V. Bonnet, F. Colledani, and P. Fraisse, "Ergonomic contribu- tion of able exoskeleton in automotive industry," International Journal of Indrustrial Ergonomics, in press.

[8] S. H. Scott, "Optimal feedback control and the neural basis of volitional motor control," Nature Reviews Neuroscience, vol. 5, no. 7, pp. 532-546, 2004.

[9] T. Flash and N. Hogan, "The coordination of arm movements: An experimentally confirmed mathematical model," The Journal of Neuroscience, vol. 5, pp. 1688-1703, July 1985.

[10] Y. Uno, M. Kawato, and R. Suzuki, "Formation and control of optimal trajectory in human multijoint arm movement," Biol. Cybern., vol. 61, no. 2, pp. 89-101, 1989.

[11] R. M. Alexander, "A minimum energy cost hypothesis for human arm trajectories," Biol. Cybern., vol. 76, pp. 97-150, Feb. 1997.

[12] T. Kang, J. He, and S. I. H. Tillery, "Determining natural arm configuration along a reaching trajectory," Experimental Brain Research, vol. 167 , no. 3, pp. 352-361, 2005.

[13] C. Harris and D. Wolpert, "Signal-dependent noise determines motor planning. [nature. 1998] - PubMed - NCBI," Nature, vol. 394 no. 6695 , pp. $780-784,1988$.

[14] C. L. Bottasso, B. I. Prilutsky, A. Croce, E. Imberti, and S. Sartirana, "A numerical procedure for inferring from experimental data the optimization cost functions using a multibody model of the neuromusculoskeletal system," Multibody System Dynamics, vol. 16, no. 2, pp. 123-154, 2006.

[15] K. Mombaur, A. Truong, and J. P. Laumond, "From human to humanoid locomotion—an inverse optimal control approach," Autonomos Robots, vol. 28, no. 3, pp. 369-383, 2010.

[16] B. Berret, E. Chiovetto, F. Nori, and T. Pozzo, "Evidence for composite cost functions in arm movement planning: An inverse optimal control approach," PLoS Computational Biology, vol. 7, pp. 1-18, Oct. 2011.

[17] V. Hue, Simulation de mouvement humain sur postes de travail pour le diagnostic et l'aide à la conception. $\mathrm{PhD}$ thesis, Université Toulouse II Paul Sabatier, Oct. 2008.

[18] C. R. Houck, J. A. Joines, and M. G. Kay, "A genetic algorithm for function optimization: a matlab implementation," NCSU-IE TR, vol. 95, no. 09, 1995.

[19] R. H. Byrd, J. C. Gilbert, and J. Nocedal, "A trust region method based on interior point techniques for nonlinear programming," Mathematical Programming, vol. 89, no. 1, pp. 149-185, 2000. 